\title{
Clinical Observation Reason For Exclusion From Statistics
}

National Cancer Institute

\section{Source}

National Cancer Institute. Clinical Observation Reason For Exclusion From Statistics. NCI Thesaurus. Code C119806.

A rationale for excluding a particular data point or subject from the clinical observation statistical analysis. 